International Electronic Journal of Algebra

Volume 31 (2022) 49-54

DOI: $10.24330 /$ ieja.1058399

\title{
PROPERTY OF DEFECT DIMINISHING AND STABILITY
}

\author{
Marco Antonio García Morales and Lev Glebsky \\ Received: 14 September 2020; Revised: 26 Mart 2021; Accepted: 16 September 2021
}

Communicated by Abdullah Harmanci

\begin{abstract}
Let $\Gamma$ be a group and $\mathscr{C}$ a class of groups endowed with bi-invariant metrics. We say that $\Gamma$ is $\mathscr{C}$-stable if every $\varepsilon$-homomorphism $\Gamma \rightarrow G,(G, d) \in$ $\mathscr{C}$, is $\delta_{\varepsilon}$-close to a homomorphism, $\delta_{\varepsilon} \rightarrow 0$ when $\varepsilon \rightarrow 0$. If $\delta_{\varepsilon}<C \varepsilon$ for some $C$ we say that $\Gamma$ is $\mathscr{C}$-stable with a linear rate. We say that $\Gamma$ has the property of defect diminishing if any asymptotic homomorphism can be changed a little to make errors essentially better. We show that the defect diminishing is equivalent to the stability with a linear rate.
\end{abstract}

Mathematics Subject Classification (2020): 20E26, 39B82

Keywords: Group theoretic stability

\section{Introduction}

The stability of a group $\Gamma$ (with respect to a class of groups $\mathscr{C}$ ) means that any almost-homomorphism to $\mathscr{C}$ is close to a homomorphism, see Definition 2.2. In [5] the notion of defect diminishing was introduced, see Definition 3.3 and Definition 3.4. It was shown in [5] that for some classes $\mathscr{C}$ and $\Gamma$-modules $M$ the vanishing of the second cohomology $H^{2}(\Gamma, M)$ implies the defect diminishing and that the defect diminishing implies stability.

In the present paper, we show that (under weaker assumptions) defect diminishing is equivalent to stability with a linear rate for finitely presented groups. It not only provides a more natural proof of Theorem 5.1 of [5] but clarifies the relation between defect diminishing and stability. Particularly, this implies that there are stable groups that do not have defect diminishing. Indeed, O. Becker and J. Mosheiff [2] showed that the rate of stability of $\mathbb{Z}^{d}, d \geq 2$ is polynomial but not linear (with respect to symmetric groups with normalized Hamming distance) ${ }^{1}$. On the other hand we do not know examples of $\left(U(n),\|\cdot\|_{p}\right)$-stable groups without the defect diminishing. Still, we do believe that such groups should exist. The problem is that the cohomological method is the only method available to show

\footnotetext{
${ }^{1}$ It is worth mentioning that the stability of any abelian group (with respect to symmetric groups with normalized Hamming distance) was proven by G. Arzhantseva and L. Păunescu in [1].
} 
stability in this case. But this leads to the defect diminishing and stability with linear rate.

\section{Stability}

Let $S$ be a finite set of symbols. We denote by $F(S)$ the free group on $S$. Let $R \subseteq F(S)$ be finite and $\Gamma$ be a finitely presented group $\Gamma=\langle S \mid R\rangle=F(S) /\langle\langle\mathrm{R}\rangle\rangle$ where $\langle\langle\mathrm{R}\rangle\rangle$ is the normal subgroup of $F(S)$ generated by $R$. Let $\mathscr{C}$ be a class of groups, all equipped with bi-invariant metric. Any map $\phi: S \rightarrow G$, for a group $G \in \mathscr{C}$ uniquely determines a homomorphism $F(S) \rightarrow G$ that we also denote by $\phi$.

Definition 2.1. [5] Let $G \in \mathscr{C}$ and let $\phi, \psi: S \rightarrow G$ be maps. The defect of $\phi$ is defined by:

$$
\operatorname{def}_{R}(\phi)=\max _{r \in R} d_{G}\left(\phi(r), 1_{G}\right)
$$

The distance between $\phi$ and $\psi$ is defined by:

$$
\operatorname{dist}_{S}(\phi, \psi)=\max _{s \in S} d_{G}(\phi(s), \psi(s))
$$

The homomorphism distance of $\phi$ is defined by:

$$
\operatorname{HomDist}_{S}(\phi)=\inf _{\pi \in H o m(\Gamma, G)} \operatorname{dist}_{S}\left(\phi, \pi \uparrow_{S}\right)
$$

Let $\left\langle\mathscr{C}^{S}\right\rangle=\bigcup_{G \in \mathscr{C}} G^{S}$ where $G^{S}=\{\phi: S \rightarrow G\}$, that is, $\left\langle\mathscr{C}^{S}\right\rangle$ are all possible maps $\phi: S \rightarrow G$ for $G \in \mathscr{C}$.

Definition 2.2. [6] A finitely presented group $\Gamma$ is called $\mathscr{C}$-stable if for all $\epsilon>0$ there exists $\delta>0$ such that for all $\phi \in\left\langle\mathscr{C}^{S}\right\rangle$ the inequality $\operatorname{def}_{R}(\phi)<\delta$ implies HomDist $_{S}(\phi)<\epsilon$. Let us restate it to avoid ambiguity:

$$
\forall \epsilon>0 \exists \delta>0 \forall \phi \in\left\langle\mathscr{C}^{S}\right\rangle\left(\operatorname{def}_{R}(\phi)<\delta \Rightarrow \operatorname{HomDist}_{S}(\phi)<\epsilon\right) .
$$

Remark 2.3. The stability of $\Gamma$ does not depend on the particular choice of the presentation of the group $\Gamma$ (see [1]): Tietze transformations preserve stability since the metric is bi-invariant. The stability of a group does depend on the class $\mathscr{C}$.

Interesting examples $\mathscr{C}=\left\{\left(G_{n}, d_{n}\right) \mid n \in \mathbb{N}\right\}$ are:

(1) $G_{n}=U(n)$, the group of Unitary $n \times n$ matrix. The metric $d_{n}$ is induced by the normalized Hilbert-Schmidt norm $\|A\|_{H S}=\sqrt{\frac{1}{n} \operatorname{tr}\left(A^{*} A\right)}\left(d_{n}(A, B)=\right.$ $\|A-B\|)$.

(2) $G_{n}=U(n)$, the metric $d_{n}$ is induced by the Schatten $p$-norm $\|A\|_{p}=\left(\operatorname{tr}|T|^{p}\right)^{\frac{1}{p}}$, where $|T|=\sqrt{T^{*} T}$. Note that if $p=2$ then $\|A\|_{2}=\|A\|_{\text {Frob }}$.

(3) $G_{n}=U(n)$, the metric $d_{n}$ is induced by the operator norm $\|A\|_{o p}=\sup _{\|v\|=1}\|A v\|$ also known as Schatten $\infty$-norm. 
(4) $G_{n}=\operatorname{Sym}(n)$, the symmetric group of $n$ elements. $d_{n}$ is the normalized Hamming distance: $d_{n}(\alpha, \beta)=\frac{1}{n}|\{j \mid \alpha(j) \neq \beta(j)\}|$.

2.1. Rate of stability. The rate of stability is, roughly speaking, the dependence of $\epsilon$ and $\delta$ in Definition 2.2. See [2] for details. To make this precise we define the function $D_{(S, R)}: \mathbb{R}^{+} \rightarrow \mathbb{R}^{+}$as follows:

$$
D_{(S, R)}(\delta)=\sup _{\phi \in\langle\mathscr{C} S\rangle}\left\{\operatorname{HomDist}_{S}(\phi) \mid \operatorname{def}_{R}(\phi)<\delta\right\}
$$

The function $D_{(S, R)}$ is monotone increasing and depends on the presentation of the group $\Gamma$, but we show now that this dependence is just linear.

The following lemma is a reformulation of Definition 2.2. The analogue of the lemma is used as the definition of stability in [3].

Lemma 2.4. $\lim _{\delta \rightarrow 0^{+}} D_{(S, R)}(\delta)=0$ if and only if $\Gamma$ is $\mathscr{C}$-stable.

Following O. Becker and J. Mosheiff, we define the rate stability $D_{\Gamma}$ of the group $\Gamma$ as a class of functions (see Definition 2.7).

Definition 2.5. Let $f, g:\left(0, \delta_{0}\right] \rightarrow \mathbb{R}^{+}$be monotone nondecreasing functions. Write $f \preceq g$ if $f(\delta) \leq g(C \delta)+C \delta$ for some $C>0$ and all $\delta \in\left(0, \delta_{0}\right]$ for some $\delta_{0}>0$. We define the equivalence relation $\sim$ by saying that $f \sim g$ if and only if $f \preceq g$ and $g \preceq f$ (notice that the relation $\preceq$ is reflexive and transitive). Let $[f]$ denote the class of $f$ with regard to this equivalence relation. Clearly, $\preceq$ defines a partial order on equivalence classes: $[f] \preceq[g]$ if and only if $f \preceq g$.

Note that if $f \preceq$ id then $f(\delta) \leq M \delta$ for some $M$. Here id is an identical function: $\operatorname{id}(\delta)=\delta$.

Proposition 2.6. [2] Let $\Gamma=\langle S \mid R\rangle$ be a finitely presented group. If $\Gamma=\left\langle S^{\prime} \mid R^{\prime}\right\rangle$ is another finite presentation of $\Gamma$. Then $D_{(S, R)} \sim D_{\left(S^{\prime}, R^{\prime}\right)}$.

Definition 2.7. Let $\Gamma=\langle S \mid R\rangle$ be a finitely presented group. The rate stability $D_{\Gamma}$ of the group $\Gamma$ is the equivalence class $D_{\Gamma}=\left[D_{(S, R)}\right]$.

Proposition 2.6 implies that the rate of stability $D_{\Gamma}$ of the finitely presented group $\Gamma$ does not depend on the presentation of $\Gamma$.

By the definition of $\sim$ the rate of stability $D_{\Gamma}$ of a group $\Gamma$ can not be faster then linear. The following lemma shows that it is not just by definition of $\sim$ but rather a natural phenomenon for non-free groups. 
Lemma 2.8. [2] Let $\Gamma=\langle S \mid R\rangle$ be a finitely presented group with $R \neq \emptyset, R \neq\left\{1_{\Gamma}\right\}$ and $\mathscr{C}$ is the class of symmetric groups with the normalized Hamming distance. Then there exists $C>0$ and $\delta_{0}>0$ such that $C \delta \leq D_{(S, R)}(\delta)$ for all $\delta \in\left(0, \delta_{0}\right]$.

By O. Becker and J. Mosheiff [2] if $\mathscr{C}$ is symmetric group with Hamming distance and $d=2,3,4, \ldots$ then $\left[\delta^{1 / b}\right] \preceq D_{\mathbb{Z}^{d}} \preceq\left[\delta^{1 / c}\right]$ for any $b<2$ and some $c=c_{d}$, depending on $d$.

\section{Property of defect diminishing}

In this section we give the definition of the property of defect diminishing and a proof of the main theorem.

Definition 3.1. An ultrafilter $\mathcal{U}$ on $\mathbb{N}$ is a collection of subsets of $\mathbb{N}$, such that:

(i) $A \in \mathcal{U}$ and $A \subset B$ implies $B \in \mathcal{U}$,

(ii) $A, B \in \mathcal{U}$ implies $A \cap B \in \mathcal{U}$,

(iii) $A \notin \mathcal{U}$ if, and only if $\mathbb{N} \backslash A \in \mathcal{U}$.

We say that $\mathcal{U}$ is non-principal if $\{n\} \notin \mathcal{U}$ for every $n \in \mathbb{N}$. The existence of non-principal ultrafilters on $\mathbb{N}$ is ensured by the axiom of choice. We fix a nonprincipal ultrafilter $\mathcal{U}$ on $\mathbb{N}$. Given a bounded sequence $\left(x_{n}\right)_{n \in \mathbb{N}}$ of real numbers we denote the limit along the ultrafilter by $\lim _{n \rightarrow \mathcal{U}} x_{n} \in(-\infty, \infty)$. Formally, the limit is the unique $x \in \mathbb{R}$ such for all $\epsilon>0$ we have $\left\{n \in \mathbb{N}:\left|x_{n}-x\right|<\epsilon\right\} \in \mathcal{U}$. For more information on ultrafilters and ultralimits see [4] appendix B.

We will use the notation Landau, let $\left(x_{n}\right)_{n \in \mathbb{N}}$ and $\left(y_{n}\right)_{n \in \mathbb{N}}$ be two sequences of positive real numbers, we denote by $x_{n}=O_{\mathcal{U}}\left(y_{n}\right)$ if there exists $C>0$ such that $\left\{n \mid x_{n} \leq C y_{n}\right\} \in \mathcal{U}$. We denote by $x_{n}=o_{\mathcal{U}}\left(y_{n}\right)$ if there is a third sequence of positive real numbers $\varepsilon_{n}$ such that $\lim _{n \rightarrow \mathcal{U}} \varepsilon_{n}=0$ and $x_{n}=\varepsilon_{n} y_{n}$.

Definition 3.2. [5] A sequence of maps $\phi_{n}: S \rightarrow G_{n}$, for $\left(G_{n}, d_{n}\right) \in \mathscr{C}$ is called an asymptotic homomorphism to $\mathscr{C}$ if

$$
\lim _{n \rightarrow \mathcal{U}} \operatorname{def}_{R}\left(\phi_{n}\right)=0 .
$$

Definition 3.3. Let $\phi_{n}: S \rightarrow G_{n}$ with $G_{n} \in \mathscr{C}$ be an asymptotic homomorphism, we say that an asymptotic homomorphism $\phi_{n}^{\prime}: S \rightarrow G_{n}$ diminishes the defect of $\left(\phi_{n}\right)_{n \in \mathbb{N}}$ if:

(a) $\operatorname{dist}_{S}\left(\phi_{n}, \phi_{n}^{\prime}\right)=O_{\mathcal{U}}\left(\operatorname{def}_{R}\left(\phi_{n}\right)\right)$,

(b) $\operatorname{def}_{R}\left(\phi_{n}^{\prime}\right)=o_{\mathcal{U}}\left(\operatorname{def}_{R}\left(\phi_{n}\right)\right)$. 
We say that $\left(\phi_{n}\right)_{n \in \mathbb{N}}$ has the property of defect diminishing if there is an asymptotic homomorphism $\left(\phi_{n}^{\prime}\right)_{n \in \mathbb{N}}$ that diminishes the defect of $\left(\phi_{n}\right)_{n \in \mathbb{N}}$.

Definition 3.4. The group $\Gamma$ has the property of defect diminishing (with respect to $\mathscr{C}$ ) if every asymptotic homomorphism to $\mathscr{C}$ has the property of defect diminishing.

Theorem 3.5. Let $\Gamma=\langle S \mid R\rangle$ be a finitely presented group and $\mathscr{C}$ a class of groups such that each $(G, d) \in \mathscr{C}$ is a complete metric space. Then the group $\Gamma$ has the property of defect diminishing if and only if $D_{(S, R)} \preceq \mathrm{id}$.

Corollary 3.6. Let $\Gamma$ be a finitely presented group and $\mathscr{C}$ a class of groups such that each $(G, d) \in \mathscr{C}$ is a complete metric space. The group $\Gamma$ has the property of defect diminishing if and only if $D_{\Gamma} \preceq[\mathrm{id}]$.

Corollary 3.7. The property of defect diminishing does not depend on the particular choice of the presentation of the group $\Gamma$.

Proof. If $\mathscr{C}$ is a class of groups such that each $(G, d) \in \mathscr{C}$ is a complete metric space, the proof follows from the Proposition 2.6 and Theorem 3.5. General case may be proved directly similarly to Proposition 2.6.

For the proof of Theorem 3.5 we need the following proposition.

Proposition 3.8. If $\Gamma=\langle S \mid R\rangle$ has the property of defect diminishing then there exists $M, \varepsilon \in \mathbb{R}^{+}$such that for all $G \in \mathscr{C}$ and $\phi \in G^{S}$ with $\operatorname{def}_{R}(\phi)<\epsilon$ there exists $\psi \in G^{S}$ such that:

(1) $\operatorname{def}_{R}(\psi)<\frac{1}{2} \operatorname{def}_{R}(\phi)$.

(2) $\operatorname{dist}_{S}(\phi, \psi)<M \operatorname{def}_{R}(\phi)$.

Proof. Suppose that the conclusion of the proposition is false. Then for every $n \in \mathbb{N}$ there is $\phi_{n} \in\left(G_{n}\right)^{S}$ with $G_{n} \in \mathscr{C}$ and $\operatorname{def}_{R}\left(\phi_{n}\right)<\frac{1}{n}$, such that every $\psi \in\left(G_{n}\right)^{S}$ with $\operatorname{def}_{R}(\psi)<\frac{1}{2} \operatorname{def}_{R}\left(\phi_{n}\right)$ satisfies $\operatorname{dist}_{S}\left(\phi_{n}, \psi\right) \geq n \operatorname{def}_{R}\left(\phi_{n}\right)$.

So we have an asymptotic homomorphism $\left(\phi_{n}\right)_{n \in \mathbb{N}}$ that does not have the property of defect diminishing. Therefore, $\Gamma$ does not have the property of defect diminishing.

Proof of Theorem 3.5. Suppose that $D_{(S, R)} \preceq$ id, that is, there exists $M>0$ and $\delta_{0}>0$ such that $\forall 0<\delta<\delta_{0}$ we have that $D_{(S, R)}(\delta)<M \delta$. Let $\left(\phi_{n}\right)_{n \in \mathbb{N}}$ be an asymptotic homomorphism and $\epsilon_{n}=\operatorname{def}_{R}\left(\phi_{n}\right)$. By the definition of asymptotic homomorphism $\lim _{n \rightarrow \mathcal{U}} \epsilon_{n}=0$. Let $X=\left\{n \mid \epsilon_{n}<\delta_{0}\right\}$. For $n \in X$ we have that 
$\operatorname{HomDist}_{S}\left(\phi_{n}\right)<M \epsilon_{n}$ by Definition 2.1 and there is a $\pi_{n} \in \operatorname{Hom}\left(\Gamma, G_{n}\right)$ that complies $\operatorname{dist}_{S}\left(\phi_{n}, \pi_{n} \uparrow_{S}\right)<M \operatorname{def}_{R}\left(\phi_{n}\right)$. Define $\phi_{n}^{\prime}=\pi_{n}$ for $n \in X$ and $\phi_{n}^{\prime}=\phi_{n}$ for $n \notin X$. Then $\phi_{n}^{\prime}$ diminishing the defect of $\phi_{n}$ as $X \in \mathcal{U}$.

Suppose that the group $\Gamma$ has the property of defect diminishing. We apply Proposition 3.8. Let $M, \varepsilon \in \mathbb{R}^{+}$be as in Proposition 3.8. Let $\phi \in G^{S}$ be with $\operatorname{def}_{R}(\phi)<\varepsilon$. Inductively we may construct a sequence of maps $\phi_{j} \in G^{S}, \phi_{0}=\phi$, such that $\operatorname{def}_{R}\left(\phi_{j}\right)<\frac{1}{2} \operatorname{def}_{R}\left(\phi_{j-1}\right)<\frac{\varepsilon}{2^{j}}$ and $\operatorname{dist}_{S}\left(\phi_{j}, \phi_{j-1}\right)<M \operatorname{def}_{R}\left(\phi_{j-1}\right)<$ $M \operatorname{def}_{R}(\phi) \frac{1}{2^{j-1}}$. It follows that $\left(\phi_{n}\right)_{n \in \mathbb{N}}$ is a Cauchy sequence. The Cauchy sequence $\left(\phi_{n}\right)_{n \in \mathbb{N}}$ of points in $G^{S}$ has a limit that is also in $G^{S}$, this limit we denote by $\phi_{\infty}$. We can check that $\phi_{\infty}$ is a homomorphism and $\operatorname{dist}_{S}\left(\phi, \phi_{\infty}\right)<2 M \operatorname{def}_{R}(\phi)$. It follows that $D_{(S, R)}(\delta)<2 M \delta$ for $\delta<\varepsilon$. Therefore, $D_{(S, R)} \preceq$ id.

\section{References}

[1] G. Arzhantseva and L. Păunescu, Almost commuting permutations are near commuting permutations, J. Funct. Anal., 269(3) (2015), 745-757.

[2] O. Becker and J. Mosheiff, Abelian groups are polynomially stable, Int. Math. Res. Not. IMRN, 20 (2021), 15574-15632.

[3] M. Burger, N. Ozawa and A. Thom On Ulam stability, Israel J. Math., 193(1) (2013), 109-129.

[4] V. Capraro and M. Lupini, Introduction to Sofic and Hyperlinear Groups and Connes' Embedding Conjecture, Lecture Notes in Mathematics, vol. 2136, Springer, Cham, 2015.

[5] M. De Chiffre, L. Glebsky, A. Lubotzky and A. Thom, Stability, cohomology vanishing, and nonapproximable groups, Forum Math. Sigma, 8 (2020), Paper No. e18 (37 pp).

[6] A. Thom, Finitary approximations of groups and their applications, In Proceedings of the International Congress of Mathematicians-Rio de Janeiro 2018, vol. III. Invited Lectures, World Sci. Publ., Hackensack, NJ, (2018), 1779-1799.

Marco Antonio García Morales and Lev Glebsky (Corresponding Author)

Instituto de Investigación en Comunicación Óptica, UASLP

Av. Karakorum 1470, Lomas 4a sección

San Luis Potosí, S.L.P, México

emails: marco13_760@hotmail.com (M.A.G. Morales)

glebsky@cactus.iico.uaslp.mx (L. Glebsky) 